\title{
Benefits of a DGH Nutrition Team are sustained over a 9 year period
}

\author{
D. Lloyd, C. Best, H. Hitchings and H. Gordon \\ Royal Hampshire County Hospital, Winchester, SO22 5DG, UK
}

A recent NCEPOD report has suggested considerable shortfalls in the provision of parenteral nutrition (PN) in UK hospitals ${ }^{(1)}$. NICE guidance on nutrition support in hospitals recommends that all acute hospital trusts should have a multidisciplinary nutrition support team (NST) to provide advice on nutrition support and especially the provision of $\mathrm{PN}^{(2)}$. Despite this, such teams are not active in many acute trusts. Our medium sized DGH (serving a population of 270,000) established a NST consisting of a consultant gastroenterologist, specialist nurse, dietician and pharmacist in 2001. The aim of this study was to review the activity of the NST and evaluate the clinical and financial impact on provision of PN.

A database of all patients who have been referred to the NST for provision of PN has been maintained since the NST was established in 2001; data are collected on an annual basis from April to April. This database was reviewed to obtain data on annual number and source of referrals, percentage who received PN, duration of PN, and wastage of PN bags. Pharmacy records were interrogated to obtain data on annual PN costs. Data were reviewed for a nine year period from April 2001 to April 2010.

The annual number of patients referred for PN ranged from 61 to 87 with no apparent trend over the review period. Percentage of patients accepted ranged from $83 \%$ to $95 \%$. Approximately half of patients (range $48 \%$ to $57 \%$ ) were referred from a critical care (HDU/ ICU) setting and the majority of patients referred were under the care of GI surgical teams. Median duration of PN ranged from 6 to 14 days with no apparent trend over the review period. Wastage of PN bags appeared to fall over the 9 year review period with a mean of 44 bags per year wasted over the 3 year period from April 2001 to April 2004 compared to 22 bags per year over the period from April 2007 to April 2010. Annual cost of PN was $£ 51,600$ in the year prior to inception of the NST; in the subsequent year the annual cost of PN fell to $£ 26,424$ (47\% reduction) and was maintained between $£ 18,120$ and $£ 39,780$ over subsequent years.

The majority of patients receiving PN in our DGH are surgical patients with short lived reversible (type 1) intestinal failure. Since inception of the NST referrals for PN have remained constant as has duration of treatment. However compared to the year prior to the establishment of the NST there has been a sustained reduction in the cost of PN. Reasons for this include reduction of inappropriate provision of PN, reduced duration of PN and reduced wastage of PN bags. This study confirms the cost effectiveness of NSTs in a DGH setting.

1. A mixed bag. An enquiry into the care of hospital patients receiving parenteral nutrition. NCEPOD 2010

2. Nutrition support for adults, oral nutrition support, enteral tube feeding and parenteral nutrition. National Collaborating Centre for Acute Care 2006. 